\title{
TOXEMIAS GRAVIDICAS
}

Conferencia dictada en el curso de post-graduados de la Universidad de América. 1956.

\section{Doctor Miguel A. Fernández-Bastidas (*)}

Las Toxemias Gravídicas, punto de la mayor trascendencia en nuestro mundo médico actual, y que últimamente ha figurado en el temario de no pocos Congresos Regionales o Internacionales cie la especialidad en dos Convenciones de Obstetricia y Ginezología del país, y de nuevo en a próxima que tendrá lugar en el año 1957 en la ciudad de Manizales; necesariamente debe llamar la atención de todos los que ejercemos la Obstetricia, puesto que contribuír a su estudio, es contribuír a solucionar el grave problema de la mortalidad materna en nuestra patria por esta causa.

De esta manera tenemos que la incidencia de Toxemia en Bogotá es de $1,22 \%$ a $0,99 \%$, la primera en clientela hospitalaria y a segunda en pacientes de un nivel social algo más elevado, y con cuidados prenatales más estrechos, como acontece con las pacientes seguidas por nosotros en el Seguro Social. Hasta 1953, ocupaba en nuestra Clínica con el $41,9 \%$ el primer lugar entre las causas puramente obstétricas de mortalidad materna sobre el número total de muertes; cifra que felizmente hoy hemos logrado bajar al $6,66 \%$ como lo atestiguan nuestras últimas publicaciones. (1).

Considerando como toxemias una o más enfermedades de aparición durante el embarazo o el puerperio inmediato y caracterizadas por edema (o marcado aumento de peso), proteinuria, hipertensión, convulsiones y coma, con alteraciones funcionales concomitantes renales, cerebrales o visuales, y que en la genera-

(*) Miembro honorario de la Academia de Medicina de San Paulo (Brasil). Ex-Jefe del Departamento de Toxemias de la Clínica $1^{\text {o }}$ de Mayo. Coordinador Nacional del Estudio de Toxemias. 
lidad de los casos hacen su aparición entre la vigésima cuarta y trigésima semana de la gestación, haciéndose evidente en no pocos casos durante el trabajo del parto o pocas horas después de éste; trataremos en seguida de comentar algo en relación con la nueva clasificación adoptada, el diagnóstico, las bases fisiopatológicás del tratamiento, y por último la conducta a seguir una vez hecho dicho diagnóstico.

Clasificación (2). Basada ésta ante todo sobre un diagnóstico preciso, y englobando únicamente entidades de una fisiopatología común, tenemos que es la siguiente:

TOXEMIA I-Toxemia Aguda $\left\{\begin{array}{l}\text { Preeclampsia } \\ \text { Eclampsia }\end{array}\left\{\begin{array}{l}\text { leve } \\ \text { grave }\end{array}\right.\right.$

TOXEMIA II.-Enfermedad crónica hipertensiva

(hipertensión esencial)

a) Asociada a una toxemia aguda.

b) No asociada a toxemia aguda

La forma aguda se identificaría por su aparición súbita, casi siempre en el último trimestre y sin ningún antecedente de hipertensión o lesión renal. Comprende la preclampsia en un primer estado, y la eclampsia su estado más avanzado. La forma crónica, liamada también hipertensión esencial, no siempre patrimonio único de la embarazada, colocada en la clasificación, por presentar siempre un estado de agravación durante el embarazo, y porque aun sin tener la paciente una lesión renal inicial, se ve iniluenciada desfavorablemente por la preñez.

Esta clasificación, que a ojos vista se aparta fundamentalmente de la tan difundida del American Comittee on Maternal Health de los años 1937 y 1939, en la que se incluía la hiperemesis gravídica, la atrofia amarilla del higado etc. y en la que figuraban "toxemias no clasificadas" tiene para el médico práctico la enorme ventaja no sólo de su sencillez y precisión y de tener una base firme anatomoclínica reconocida, sino por haber sido adoptada oficialmente como la única en la Segunda Convención cie Obst. y Ginec. reunida en Cali el año pasado.

Díagnóstico.-Dieckmann opina que aproximadamente la mitad de las pacientes que se dice padecen de la llamada toxemia del embarazo tienen hipertensión de otro origen o algún tipo de 
enfermedad vásculo-renal y sólo las restantes el síndrome preeclampsia-eclampsia; y agrega que la toxemia aguda no produce alteraciones anatomopatológicas del tipo vásculo-renal en la embarazada a pesar de ésta poseer señales evidentes de hipertensión, pulso rápido, respiración agitada, oliguria, síntomas de falla cardíaca, convulsiones, hemoconcentración, etc., signos y síntomas que revelan ef grave estado en que se encuentra la enferma (3).

Hoy día hay tendencia a pensar, en contra de lo dicho anteriormente, que a pesar de no hacerse patente en los primeros meses los signos de la toxemia aguda, ésta inicia su aparición antes de la trigésima semana de la gestación; asegurándose que aun sin la aparición de edemas la embarazada puede estar reteriendo agua en sus tejidos, y que la única manera de demostrarlo es el exagerado aumento de peso observado en la gestante que pone al médico sobre aviso, siendo de tánta importancia como el control de pulso, de tensión, y el uranálisis al efectuar un apropiado control pre-natal.

Al examinar por primera vez una embarazada nunca debemos de supervalorar, el hallazgo de tensión alta de edemas o de proteinuria, tratando de interpretarlos a priori como producidos por una toxemia pues obrando de esta manera nunca podremos hacer un diagnóstico acertado. En relación con los datos tensionales por ejemplo, no debemos olvidar que los límites de la presión sanguínea normal varían con la edad, dando un límite de hipertensión en la tercera década de 140/90, y en la cuarta aproximadamente de $150 / 98$. De la misma manera la valoración e interpretación del signo proteinuria, exige conocer desde la forma como fue obtenida la muestra de orina (por cateterismo o no), ia cantidad de ésta en las 24 horas, y la valoración del sedimento nallado en relación con el hallado en la embarazada normal. Si la proteinuria ha sido demostrada como tal, hacer el diagnóstico ciferencial entre las causas que de causa renal o extrarrenal la puedan producir. Al hallar igualmente una paciente edematizada. pensar en la posibilidad de que este edema sea causado por alteraciones venosas tan frecuentes en los miembros inferiores de este tipo de enfermas, por alteraciones metabólicas, o por anemia, cardiopatía, neuropatías, etc., o bien si no es muy marcada, sin interpretación patológica en el curso de un embarazo normal. Por último volviendo al signo hipertensión, su hallazgo no debe hacernos olvidar que un embarazo puede estar complicado con un feocromocitoma, una nefroesclerosis; o bien el alza de presión 
ser ocasionada simplemente por miedo, excesivo trabajo muscular (trabajo del parto) o bien a causa de una acción medicamentosa (pituitrina).

De manera que en presencia de uno, dos o tres de los signos ya largamente descritos como inequívocos de toxemias; sin un examen detenido de la paciente sólo obtendremos una presunción de ésta, siendo el aboratorio y los exámenes y controles complementarios los únicos medios que nos darán un fundamento cierto para sentar el diagnóstico (4).

¿Cuántas veces se nos ha relatado historias clínicas de pacientes gestantes en que las convulsiones y el coma se atribuyeron sin mayor examen, a una crisis eclámptica? Pero en las que no se llegó a descartar la posibilidad de una encefalopatía hipertensiva, una uremia, una intoxicación anestésica durante el parto (óxido nitroso, ciclopropano, etileno) una reacción a drogas eceleradoras (iel parto (pituitrina) o bien una histeria, epilepsia, alcalosis, crisis hipoglicémica (por dosis excesivas de insulina) tumor o abceso cerebral, etc.?

En otros casos el diagnóstico de "preeclampsia sobre agregada" en pacientes con nefropatía, demostrada antes del embarazo, y que durante el cual complican su alteración inicial, tratan de hacer aparecer como toxemias casos completamente ajenos a ellas.

El antecedente de Toxemia en los embarazos anteriores, al contrario de lo comúnmente admitido no tiene mayor valor $\mathrm{y}$ hoy es aceptado que una paciente cuyo primer embarazo se vio complicado con toxemia, (diagnóstico exacto) no tiene por qué en ausencia de lesión renal o hepática, repetir fatalmente sus crisis toxémicas en los embarazos subsiguientes.

Así pues, el dagnóstico clínico de Toxemia sólo debe hacerse analizando en conjunto toda la anamnesia, prestando especial atención a la edad de la paciente, la paridad, la época de aparición y duración de la hipertensión, los edemas, y las cantidades anormales de proteína en la orina. La edad por ejemplo más frecuente hallada por nosotros fue entre los 20 y los 23 años, siendo de anotar que en general es la edad en que nuestras mujeres tienen su primer embarazo y parto (5). En cuanto a la paridad, ya dijimos ser más frecuente su aparición en las primigrávicias, posiblemente por existir un mayor tono muscular uterino y abdominal que al producir un aumento de la presión venosa de las extremidades inferiores y del útero llega a ser un factor predis- 
ponente de isquemia uterina, tal como sucede en no pocos casos de polidramnios y embarazo múltiple complicados con toxemia.

En cuanto al tiempo de aparición de la hipertensión generalmente es tardío, alrededor del séptimo $u$ octavo mes de la gestación; de tal manera que en los casos en que ha habido una cuidadosa vigilancia prenatal, por simple observación se descarta cualquier otra causa hipertensiva preexistente, y sólo en el caso cie tratarse de una típica hipertensión esencial, puede ayudar a orientar el diagnóstico.

En resumen, solamente la atenta observación clínica de la enferma por un lapso no menor de 24 horas desde el momento en que por primera vez se han hallado los signos de presunción, colocándola en completo reposo y dieta apropiada, de preferencia en un medio hospitalario, tiempo durante el cual se practican los exámenes de laboratorio que detallaremos más adelante, podemos fundamentar un diagnóstico preciso. Gracias a esta norma de conducta hemos logrado descartar no pocos diaginósticos hechos de manera apresurada, que de otra manera hubieran influído sobre un tratamiento empírico, y en no pocas ocasiones completamente inútil (6).

En cuanto a la ayuda dada por el laboratorio, y los exámenes complementarios; el examen de orina con muestra extraída con sonda, informará la existencia de proteinuria con cifras que oscilan entre 1 ar 5 y más gramos por litro, y un sedimento en especial en los casos no muy avanzados negativo para cilindros granulosos. El examen de química sanguínea con cifras de N. P. N. dentro de límites normales (de 25 a 40 mgrs. \%) aun en casos de eclampsia grave, descartándose la existencia de lesión renal avanzada; proteínas sanguíneas normales en los casos leves, y aumentadas en los casos de hemoconcentración; y generalmente cifras de uricemia algo aumentadas. Al examen de cuadro hemático, un aumento de hematocrito y hemoglobina nos dará una noción del grado de hemoconcentración en los casos graves. Por último el examen de fondo de ojo con imágenes inconfundibles de espasmo arteriolar retiniano, edema papilar y en casos graves hemorragias retinianas, que tienen como característica especial el ser reversible y desaparecer con la mejoría del estado toxémico. En los casos en que después de toda esta serie de pruebas se sospecha una lesión renal aconsejamos la práctica de pruebas de funcionamiento renal, que serán presentadas en detalle en la conferen- 
cia que sobre enfermedad renal y embarazo dictará en este curso el doctor B. Calderón (7).

Tratamiento.-En cuanto hace a este importante capitulo de las Toxemias, por tratarse de una entidad de etiología bastante discutida aun hoy día, prima ante todo un tratamiento sintomático. Por esta razón, y con el único objeto de hacer lo más práctica que sea posible esta actualización sobre el tema, nos contentaremos con exponer las bases esenciales fisiopatológicas, sobre las cuales hemos fundamentado la terapéutica en nuestro Servicio especializado y cuyas conclusiones aparecen en la ponencia que sobre el tema presentamos en la última Convención cie Obstetricia y Ginecología reunida en Cali el año pasado. De esta manera veremos cómo tratamos de correlacionar el signo hipertensión con la medicación hipotensora, la proteinuria y la retención anormal de líquidos con la medicación derivativa y la cieta hipoclorurada, y por último el estado convulsivo con el єmpleo de drogas neuroplégicas y sedantes del sistema nervioso central.

Analicemos en primer término el signo hipertensión en general; y veremos que su aparición es debida:

a) A un aumento en el volumen minuto cardíaco, tal como sucede en la embarazada normal, en la que es frecuente hallar gran inestabilidad tensional, o en pacientes hiperemotivas en las cuales la sola presencia del médico puede ser causa transitoria suficiente de aumento en las cifras tensionales.

b) O a un aumento en el volumen sanguíneo, tal como sucede en los casos de plétora sanguínea.

c) A una disminución de la elasticidad arteriolar, tal como ocurre con la arterioesclerosis.

d) A un aumento del índice de viscosidad sanguínea (normal 3,6 a 5,4) generalmente atribuíble a un estado de deshidratación real o aparente (paso de agua a los espacios intersticiales) y haciendo parte del sindrome de hemoconcentración.

e) O bien a una disminución del calibre arteriolar de origen espasmódico, y que dará hipertensiones de cifras oscilantes, que como es de suponerlo desaparecen al dejar de actuar la causa desencadenante.

Entre estas cinco causas enumeradas, dos son las de tenerse en cuenta en la paciente toxémica, una primordial la disminución del calibre arteriolar por espasmo (hecho completamente 
ciemostrado), y otra secundaria el aumento de viscosidad sanguínea, y que se halla presente solamente en los casos de toxemia aguda y grave y demostrable por cifras altas de hematocrito, de hemoglobina con poliglobulia (8).

Después de los estudios de hipertensión experimental llevados a cabo por Green entre otros y su correlación con la paciente toxémica por Garber, tenemos: que dos son los factores causantes de hipertensión en una toxemia, el factor humoral (o reno-váscuio-humoral-endocrino) y el factor neurogénico; e! primero como su nombre lo indica estaría constituido por la ya conocida renina e hipertensina (Houssay), los factores vasoconstrictor y vasopresor (V. E. M. y V. D. M. estudiados por Sweifach y Shorr) y el factor endocrino hipofisario constituído por el A. C. T. H. (9). En cuanto al factor neurogénico comandado por sistema regulador aórtico y carotideo se manifiesta al suprimirse su acción reguladora por una causa que altere su mecanismo (10).

De esta manera tenemos que en la preeclampsia grave y la enfermedad hipertensiva en que hay predominio del factor humoral las drogas hipotensoras de e'ección son el Veratrum y sus derivados así como también la clorpromazina. Y en los casos de hipertensión de origen neurogénico, presente en casos leves y de manera transitoria en la embarazada normal (necesidad del reposo en observación por 24 horas) los barbitúricos, el reposo, la dieta pobre en ión sodio, y en los casos reacios la medicación por medio de hidrazinoftalazina (Apresolina), de reserpina, o en casos especiales la combinación del Veratrum (droga de acción rápida) con la reserpina (droga de acción lenta).

Siguiendo con el segundo gran signo de Toxemia, aunque el más precoz en su aparición, la proteinuria, término más preciso que el de albuminuria para expresar la anormal eliminación de proteinas por el riñón, tenemos que al igual que la hipertensión, hoy día está demostrada una causa espasmódica arteriolar en su producción. (Chesley). Su hallazgo en la embarazada en ausencia de retención azoada y con funciones glomerular y tubular dentro de los límites normales (pruebas de función renal), rara vez se ve complicada con anuria utilizando un tratamiento apropiado a base de soluciones hiper e isotónicas de dextrosa en agua, tal como hemos tenido la oportunidad de observar en nuestra Clínica, logrando en esta forma hemodilución, aumento de la ciurosis y aumento de la reserva glicogénica hepática. 
La retención de líquidos, como ya lo señalamos al hablar del diagnóstico, nunca la valoramos únicamente por la aparición de edemas, por demás raros en los primeros estudios de la enfermedad, sino por el aumento exagerado de peso. Su patogenia está dada por el reemplazo del ión potasio por el ión sodio en el líquido intracelular, que como es sabido normalmente tiene el primero en mayor proporción; y un anormal funcionamiento corticosuprarenal. Fisiopatológicamente hablando la retención acuosa obedece a una alteración en la dinámica capilar entre la presión celoidoosmótica y la presión tisular. Con el objeto de tratar de corregir dicha falla usamos la acetozolamida (Diamox) sustancia que logra el aumento en la excreción del ión sodio y abundante diuresis, y las soluciones hipertónicas de dextrosa (sol de dextrosa al 33\% 500 c. c.) por vía endovenosa, que a más de producir hemodilución es un poderoso diurético como ya lo hemos dicho.

Por último tenemos el estado más avanzado de la Toxemia aguda y al cual no debiera llegar ninguna embarazada, si ha sido vigilada y tratada convenientemente desde la iniciación de su enfermedad, el astado convulsivo. Su patogenia está dada por una descarga nerviosa de la sustancia gris de tipo "explosivo" sobre cualquier área del sistema nervioso central teóricamente susceptible (Jackson). Para explicar su fisiopatología se han invocado multitud de teorías, entre las cuales debemos señalar: a) la teoría irritativa, que presupone la acción sobre el cortex de una causa inflamatoria, cicatricial, física o química; b) La teoría de "liberación de acción cortical", con supresión del control de los centros nerviosos; c) La teoría del "corto circuito", por destrucción de axones, transmitiéndose los impulsos nerviosos por una ruta más corta que la normal, y d) La teoría metabólica que explica la aparición de convulsiones por una alteración súbita en el metabolismo de la célula nerviosa, semejante a la producida por la anoxia. Con el objeto de atenuar o suprimir dicha descarga nerviosa creemos de acuerdo con nuestras observaciones que el uso de drogas tales como la clorpromazina (Largactil) a la dosis de 100 mgrs. por vía endovenosa, se logra plenamente este objeto (11). De la misma manera el uso del sulfato de magnesia en soluciones tidalizadas al $20 \%$ por vía intrarráquidea y a razón de un centigramo por kilogramo de peso, aunque aun en período experimental creemos logra por los éxitos obtenidos en no menos de quince casos, que actuar de manera electiva y 
de diferente manera a la vía endovenosa sobre la patogenia de las crisis eclámpticas (12).

Conducta.-La conducta seguida con la paciente toxémica, y en especial con la que ha llegado al ataque convulsivo, ha sido uno de los puntos más debatidos en patología obstétrica. Hoy día después de gran número de fracasos con el criterio intervencionista, en que toda enferma eclámptica era pasada a la sala de cirugía y se le practicaba una cesárea con esta sola indicación, se ha llegado a la conclusión que el tratamiento de la toxemia gravidica debe buscar ante todo una metódica y oportuna selección de la forma de parto, y que tanto la cesárea como la inducción deben llevarse a cabo horas después de haber utilizado el tratamiento médico (13).

A continuación y para terminar enumeramos los esquemas de tratamiento seguidos por nosotros en nuestra Clínica (Clínica $1^{\circ}$ de Mayo, Departamento de Estudio de Toxemias) (14) (15).

\section{I.-Preeclampsia leve.}

a) Período de observación por 24 horas con dieta y reposo;

b) Dieta hipoclorurada (2 grs. de NaCl y 1.200 calorías);

c) Sedación con fenobarbital 0,20 cada 12 horas vía I. M.;

d) Diamox 250 mgrs. por vía oral una vez al día (1 com.);

e) Controles: de tensión 2 veces al día, de peso todos los días, y cantidad de líquidos ingeridos y eliminados en veinticuatro horas.

f) Examen parcial de orina diario, química sanguínea, y hematocrito al ingreso y luégo del parto, y

g) En caso de mejoría, control prenatal estrecho ambulatorio hasta el parto.

\section{II.-Preeclampsia grave.}

a) Período de observación por 24 horas con dieta y reposo;

b) Controles de tensión, peso y líquidos;

c) Exámenes de rutina (orina, química, sanguínea, etc.).

d) Largactil 50 mgrs. disueltos en 500 c. c. de Dextrosa al $33 \%$ vía endovenosa, luégo comprimidos de 25 mgrs. cada ocho horas, y

e) Con mejoría, en embarazo de más de 8 y medio meses inducción. 


\section{III.-Eclampsia.}

a) Reposo absoluto;

b) Dieta hipoclorurada;

c) Exámenes y Controles de rutina (igual que en -I-);

d) Dextrosa al $33 \%$ en agua destilada 500 c. c. con 100 mgrs. de Largactil vía endovenosa;

e) Oxígeno inhalado;

f) En caso de persistencia de las convulsiones, sulfato de Magnesia al $20 \%$ por vía intrarraquídea a razón de un centigramo por $\mathrm{Kg}$. de peso, y

g) Nunca cesárea. Inducción una vez controlada la tensión.

\section{IV.-Hipertensión esencial.}

a) Periodo de observación de 24 horas con dieta y reposo;

b) Exámenes y controles de rutina;

c) Tratamiento de la hipertensión con Apresolina 40 mgrs. los dos primeros dias, luégo 100 mgrs. 8 dias, luégo 200 mgrs. otros 8 días.

d) Vigilancia ambulatoria hasta el momento del parto, y

e) Con gran proteinuria inducción a los 8 y medio meses.

\section{BIBLIOGRAFIA}

(1) FERNANDEZ-BASTIDAS, M. A.-“Toxemias Gravidicas". Ponencia al II Congreso Nacional de Obst. y Ginec. Cali. 16-1955 Multilith I. C. S. S.

(2) AFANAOR RUIZ J.-“Toxemias Gravídicas". Ponencia al II Congreso de Obst. y Ginec. de Cali. 9. 1955. Multilith. I. C. S. S.

(3) DIECKMANN W.- "Patología y Tratamiento de la Toxemia del Embarazo". Rev. de Obst. y Ginec. de Venezuela 15:435-1955.

(4) FERnANDEZ-Bastias, M. A.-Rev. Col. de Obst. y Ginec. 5:152. 1954.

(5) SILVA-MOJICA, C. R.-“Toxemia Gravídica”. II Congreso Obst. y Ginec. Cali. Pág. 1. Multilith. I. C. S. S.

(6) FERNANEZ-BASTIAS, M. A.-Rev. Méd. I. C. S. S. 1:147-1955.

(7) CALDERON BELISARIO.- "Función Renal durante el embarazo". Conf. Unv. de América. Rev. Col. de Obst. y Ginc. 7:431. 1956. 
(8) FERnANDEZ-BASTIDAS, M. A.-Rev. Col. de Obst. y Ginec. 7:7. 1955.

(9) GARBER S. ASSALI N., KIRSTNER, PRYSTOWSKY H. AM. J.Obst. and Gync. 60: 315-1950.

(10) GREEN D. M.- "Experimental hypertension and Clinical Practice". Amer. Practit. 5:243-1954.

(11) SILVA-MOJICA, C. R.-Rev. Col. de Obst. y Ginec. 5: 25-1953.

(12), CARRILLO, E. y BARRETO-BRUCE L.-“Toxemias Gravídicas". Ponencia II Congreso, de Obst. y Ginec. Cali. 1955. Pág. 67. Multilith. I. C. S. S.

(13) CALDERON M. BELISARIO.-“Toxemias Gravídicas". Ponencia al II Congreso d Obst. y Ginec. Cali. Cali. 78. 1955. Multilith. I. C .S. S.

(14) OFMAN J.-“Toxemia Gravídica”. Ponencia II Congreso Obst. y Ginec. Cali. Pág. 31. Multilith. I. C. S. S. Bogotá, D. E. 1955,

(15) UCROS-CUELLAR A.-“Toxemias Gravídicas". Ponencia II Congr. Obst. y Ginec. Cali. 1955. Pág. 34. Multilith. 1955. Bogotá, D. E. - I. C. S. S. 